Supplement of Hydrol. Earth Syst. Sci., 25, 2187-2198, 2021

https://doi.org/10.5194/hess-25-2187-2021-supplement

(c) Author(s) 2021. CC BY 4.0 License.

(c) (i)

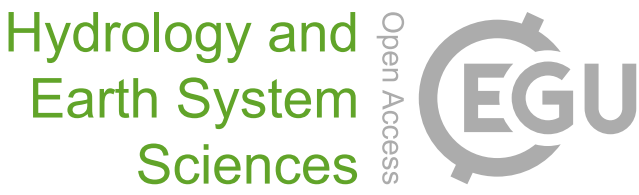

Supplement of

\title{
Technical note: Diagnostic efficiency - specific evaluation of model performance
}

Robin Schwemmle et al.

Correspondence to: Robin Schwemmle (robin.schwemmle@hydrology.uni-freiburg.de)

The copyright of individual parts of the supplement might differ from the article licence. 


\section{Supplement}
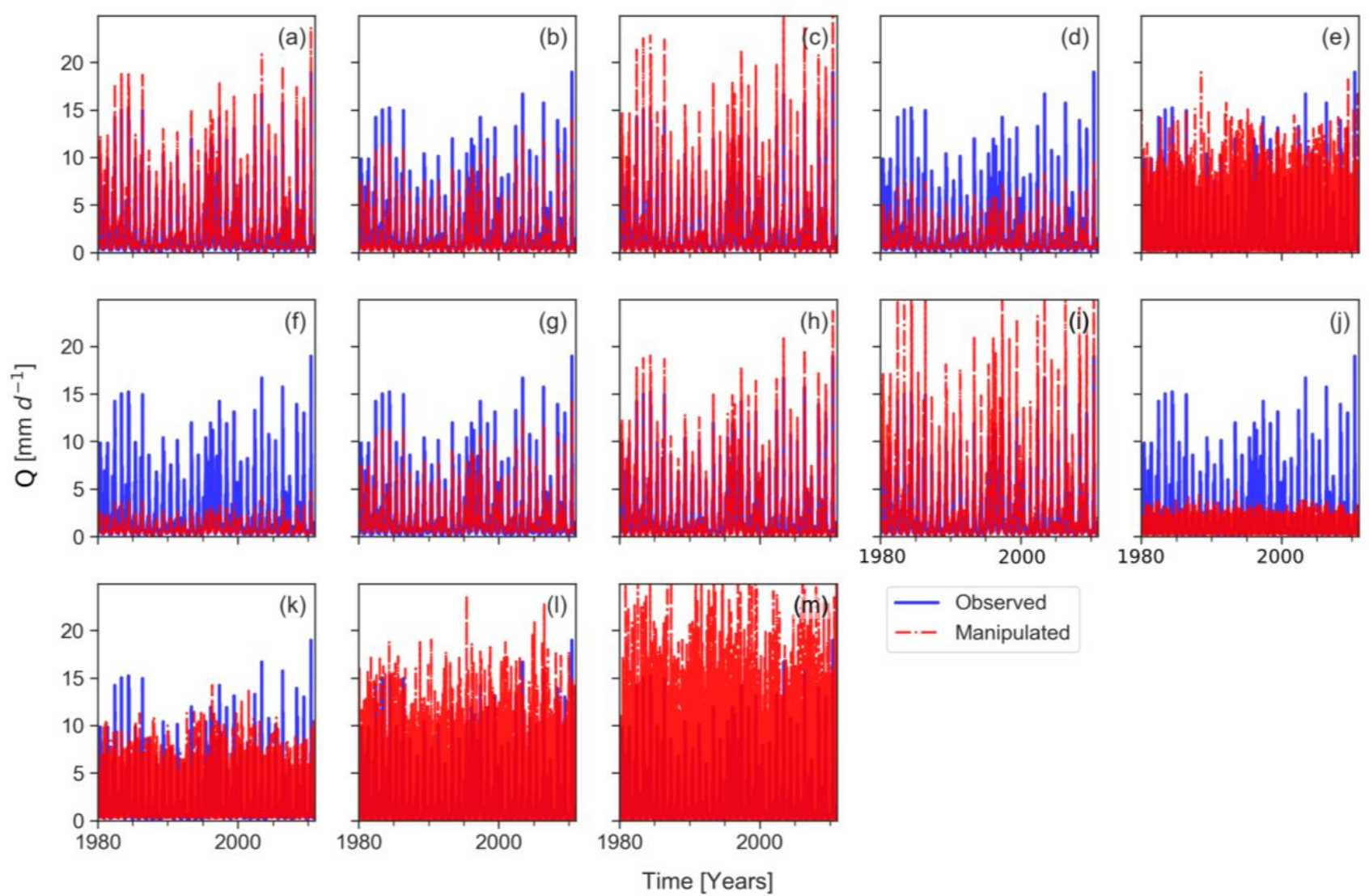

Figure S1: Observed streamflow time series and manipulated streamflow time series generated by mimicking constant errors, dynamic errors and timing errors (a-m) 

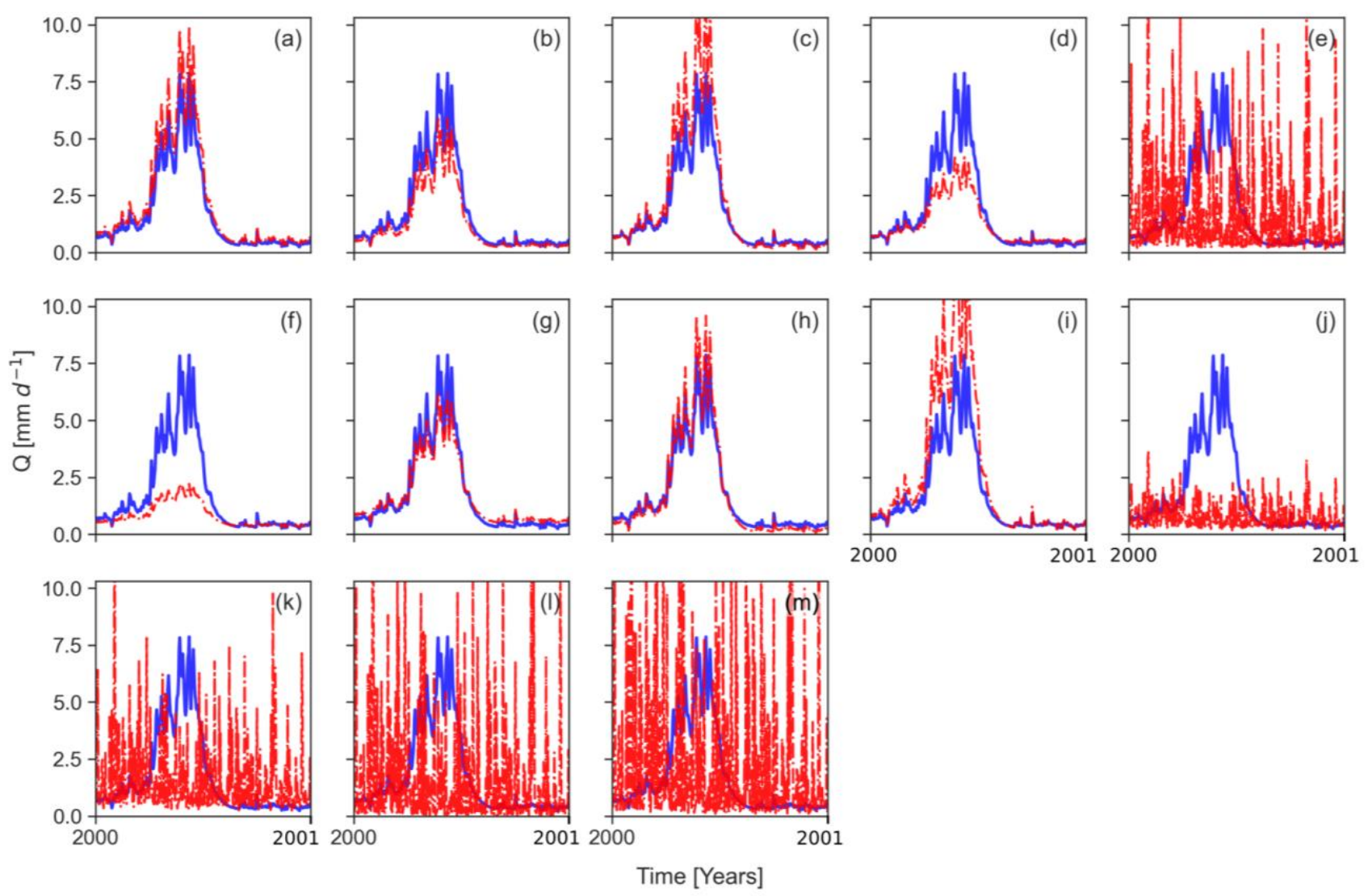

10 Figure S2: Observed streamflow time series and manipulated streamflow time series for a single year generated by mimicking constant errors, dynamic errors and timing errors (a-m) 


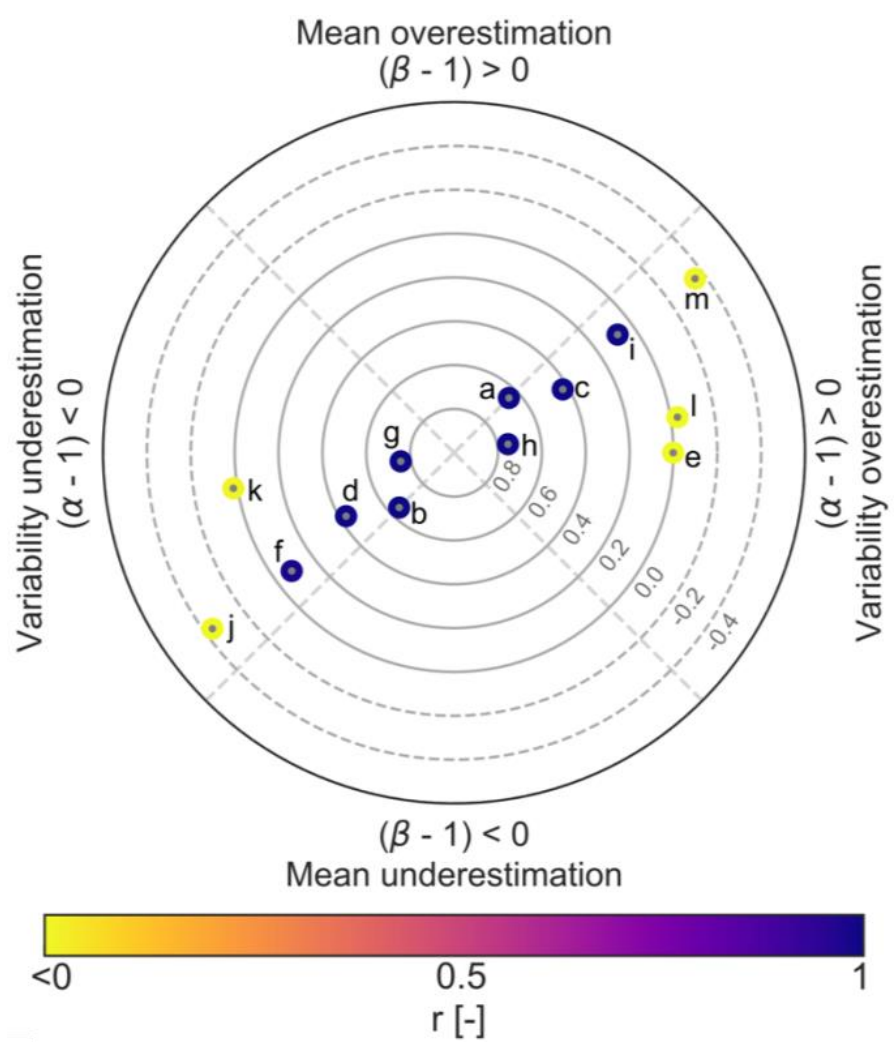

Figure S3: Polar plot of $K G E$ for manipulated time series generated by mimicking constant errors, dynamic errors and timing errors (a-m)
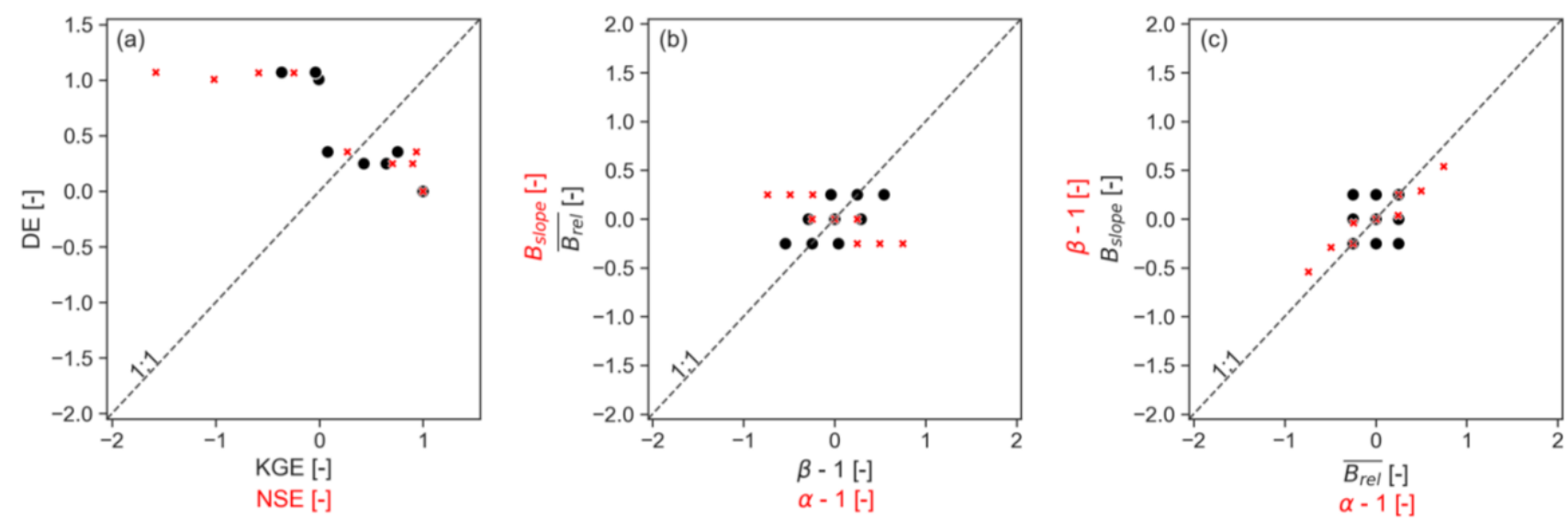

Figure S4: (a) Scatterplot to compare $D E$ with $K G E$ (black) and $D E$ with $N S E$ (red), respectively. (b) Scatterplot to compare $\overline{B_{\text {rel }}}$ with $\beta$ (black) and $B_{\text {slope }}$ with $\alpha$ (red), respectively. (c) Scatterplot to compare $\overline{B_{\text {rel }}}$ with $B_{\text {slope }}$ (black) and $\beta$ with $\alpha$ (red), respectively. Metrics are calculated for manipulated time series (see Fig. S1) 
Table S1: Comparison of $D E$ metric terms and $K G E$ metric terms for manipulated time series generated by mimicking constant errors, dynamic errors and timing errors (a-m)

\begin{tabular}{lccccccccccccc} 
& $\mathrm{a}$ & $\mathrm{b}$ & $\mathrm{c}$ & $\mathrm{d}$ & $\mathrm{e}$ & $\mathrm{f}$ & $\mathrm{g}$ & $\mathrm{h}$ & $\mathrm{i}$ & $\mathrm{j}$ & $\mathrm{k}$ & $\mathrm{l}$ & $\mathrm{m}$ \\
\hline$\overline{B_{\text {rel }}}$ & 0.25 & -0.25 & 0 & 0 & 0 & -0.25 & 0.25 & -0.25 & 0.25 & -0.25 & 0.25 & -0.25 & 0.25 \\
$\mid B_{\text {areal }}$ & 0 & 0 & 0.25 & 0.25 & 0 & 0.25 & 0.25 & 0.25 & 0.25 & 0.25 & 0.25 & 0.25 & 0.25 \\
$r$ & 1 & 1 & 1 & 1 & 0 & 0.98 & 1 & 1 & 1 & 0 & 0 & 0 & 0 \\
$B_{\text {dir }}$ & 0 & 0 & -1 & 1 & 0 & 1 & 1 & -1 & -1 & 1 & 1 & -1 & -1 \\
$B_{\text {slope }}$ & 0 & 0 & -0.25 & 0.25 & 0 & 0.25 & 0.25 & -0.25 & -0.25 & 0.25 & 0.25 & -0.25 & -0.25 \\
$B_{\text {hf }}$ & 0.13 & -0.13 & 0.13 & -0.13 & 0 & -0.25 & 0 & 0 & 0.25 & -0.25 & 0 & 0 & 0.25 \\
$B_{\text {lf }}$ & 0.13 & -0.13 & -0.13 & 0.13 & 0 & 0 & 0.25 & -0.25 & 0 & 0 & 0.25 & -0.25 & 0 \\
$B_{\text {tot }}$ & 0.25 & 0.25 & 0.25 & 0.25 & 0 & 0.31 & 0.31 & 0.31 & 0.31 & 0.31 & 0.31 & 0.31 & 0.31 \\
Ehf & 0.5 & -0.5 & 0.5 & -0.5 & 0 & -0.8 & 0 & 0 & 0.8 & -0.8 & 0 & 0 & 0.8 \\
$E_{\text {lf }}$ & 0.5 & -0.5 & -0.5 & 0.5 & 0 & 0 & 0.8 & -0.8 & 0 & 0 & 0.8 & -0.8 & 0 \\
$\beta$ & 1.25 & 0.75 & 1.29 & 0.71 & 1 & 0.46 & 0.96 & 1.04 & 1.54 & 0.46 & 0.96 & 1.04 & 1.54 \\
$\alpha$ & 1.25 & 0.75 & 1.49 & 0.51 & 1 & 0.25 & 0.76 & 1.24 & 1.75 & 0.25 & 0.76 & 1.24 & 1.75
\end{tabular}

Table S2: DE and its metric terms for the modelling example. Simulations were realised with different parameter sets (set_id).

\begin{tabular}{cccccccccccc} 
set_id & $\overline{B_{\text {rel }}}$ & $\left|B_{\text {area }}\right|$ & $r$ & $B_{\text {dir }}$ & $B_{\text {slope }}$ & $D E$ & $B_{h f}$ & $B_{\text {lf }}$ & $B_{\text {tot }}$ & $\varepsilon_{f f}$ & $\varepsilon_{\text {lf }}$ \\
\hline 05 & 0.16 & 0.32 & 0.88 & 1 & 0.32 & 0.38 & -0.07 & 0.23 & 0.31 & -0.24 & 0.76 \\
48 & 0.16 & 0.34 & 0.89 & 1 & 0.34 & 0.40 & -0.08 & 0.24 & 0.32 & -0.26 & 0.74 \\
94 & 0.11 & 0.28 & 0.89 & 1 & 0.28 & 0.32 & -0.07 & 0.18 & 0.26 & -0.28 & 0.725
\end{tabular}

Table S3: KGE (with metric terms) and NSE for the modelling example. Simulations were realised with different parameter sets (set_id).

\begin{tabular}{cccccc} 
set_id & $\beta$ & $\alpha$ & $r$ & KGE & NSE \\
\hline 05 & 0.90 & 0.79 & 0.88 & 0.74 & 0.77 \\
48 & 0.89 & 0.79 & 0.89 & 0.74 & 0.77 \\
94 & 0.90 & 0.83 & 0.89 & 0.77 & 0.78
\end{tabular}




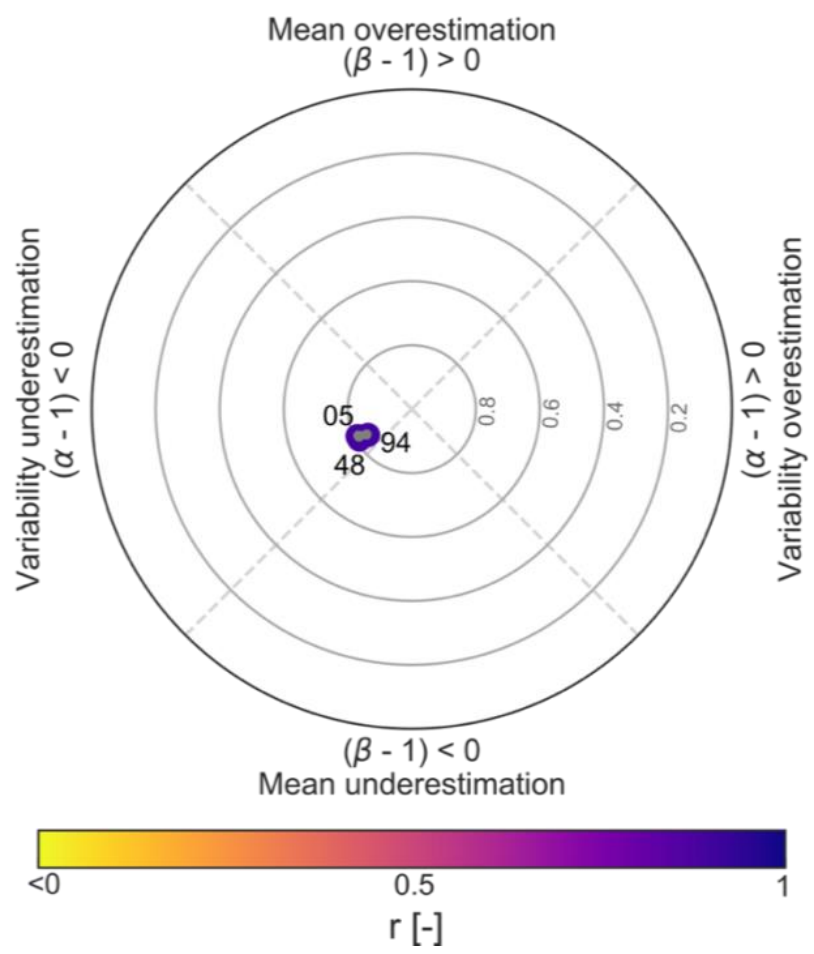

Figure S5: Polar plot of $K G E$ for modelling example. Simulations were realised with three different parameter sets (05, 48, 94; see Fig. 4). 